\title{
Astronomy Laboratory: What are we going to make today?
}

\author{
Susan Feteris and Don Hutton \\ Department of Physics, Monash University, Clayton, Vic. 3168, Australia \\ susan.feteris@sci.monash.edu.au, don.hutton@sci.monash.edu.au \\ Received 1999 October 1, accepted 2000 April 28
}

\begin{abstract}
The workshop programme of a new first year university astronomy subject with low capital costs incorporates diverse learning experiences: planisphere, time around the world, sundial, telescope, computer simulation, planetarium, spectroscope, poster, debate, night-time observatory trip. The practical activities, used to address specific learning objectives, emerged clearly as a major strength of the subject as evaluated by the mixed student cohort.
\end{abstract}

Keywords: Moon—stars: general—Sun: general—time

\section{Overview of the Subject}

First year level Astronomy attracts a diverse student body: academically strong aspiring astronauts, Star Trek junkies, academically weak students who hope that Astronomy will be easier than Physics or Chemistry, and students from faculties other than Science. Delivering a subject that fulfills the needs and expectations of students with varying motivation is no small challenge. ASP1010 'Earth to Cosmos' offered by the Department of Physics is designed to fill a demand for a first year astronomy subject, acknowledging the broad appeal of the subject matter to students entering other fields whilst also catering for those with serious ambitions in astronomy and astrophysics.

During our thirteen-week semester we deliver 39 lectures and twelve two-hour laboratory workshops. In eight of those workshops the students (in groups of 16 or less) engage in a practical activity, completing an assessment sheet at its conclusion. Four of those activities involved constructing a tool that students could take home. While initially our 'grown-up' university students were surprised at these 'cut-and-paste' activities, they involved non-trivial learning objectives and the students expressed disappointment when they did not have a 'take-home' activity in week 5 !

Besides the eight weeks of practical activities, three weeks were devoted to the preparation and presentation of posters on contemporary topics, with students working in groups of 2 or 3, and one week to a formal debate on the Cosmological Anthropic Principle, in which some students opted to take part instead of participating in the poster project. Both the poster and the debate specifically required students to work co-operatively as members of a team - an ability employers have identified as very important and which universities are now trying to explicitly address and assess.

\section{Workshop Exercises}

The eight practical activities are outlined below.

Planisphere. Introducing many concepts using a two-dimensional simulator: the ecliptic, right ascension, declination, solar and sidereal (star) time, South Celestial Pole, zenith, rotation and seasonal variation of the night sky (take-home tool ... materials cost 65\%).

Time around the world (Hutton 1998). Exploring the motion of the Moon and Sun, time differences around the world, phases of the Moon, synodic and sidereal periods of the Moon, solar and lunar eclipses, revisiting solar and sidereal time (take-home tool ... materials cost $25 \phi)$.

Equatorial sundial (Folkland \& Ward 1996). Exploring the relationship between the Sun's motion and our notion of time, challenging the common belief that $12: 00 \mathrm{pm}=$ 'midday', introducing the equation of time, reinforcing concepts developed in earlier activities (take-home tool ... materials cost 20ф)).

Planetarium (Giardini \& Hutton 1977). Using a three-dimensional simulator to move between the South Pole and the Equator and through the seasons of the year, exploring the apparent motion of the stars, Sun and Moon. Revisiting many concepts: the ecliptic, the seasons, South Celestial Pole, zenith, azimuth, and eclipses.

Telescope. Constructed on an optical bench with two or three lenses, giving students experience of the function of a simple telescope.

Spectroscope (Thompson 1996). Giving students practice in identifying emission spectra (take-home tool ... materials cost $\$ 4.50$ ).

Hertzsprung-Russell diagrams. Giving students experience in interpreting star data.

'Contemporary Laboratory Experiences in Astronomy' simulation-redshift. ${ }^{*}$ Exposing students to analysis tools used by astrophysicists to determine the Hubble constant.

The take-home activities in particular proved extremely popular. Students were able to use them when appropriate (e.g. on a clear night) for reinforcement of the concepts learned. We have developed some educationally valuable yet cheap practical activities that enhanced our first year astronomy subject. We have had low capital costs associated with the introduction of this new subject and modest continuing materials costs. 


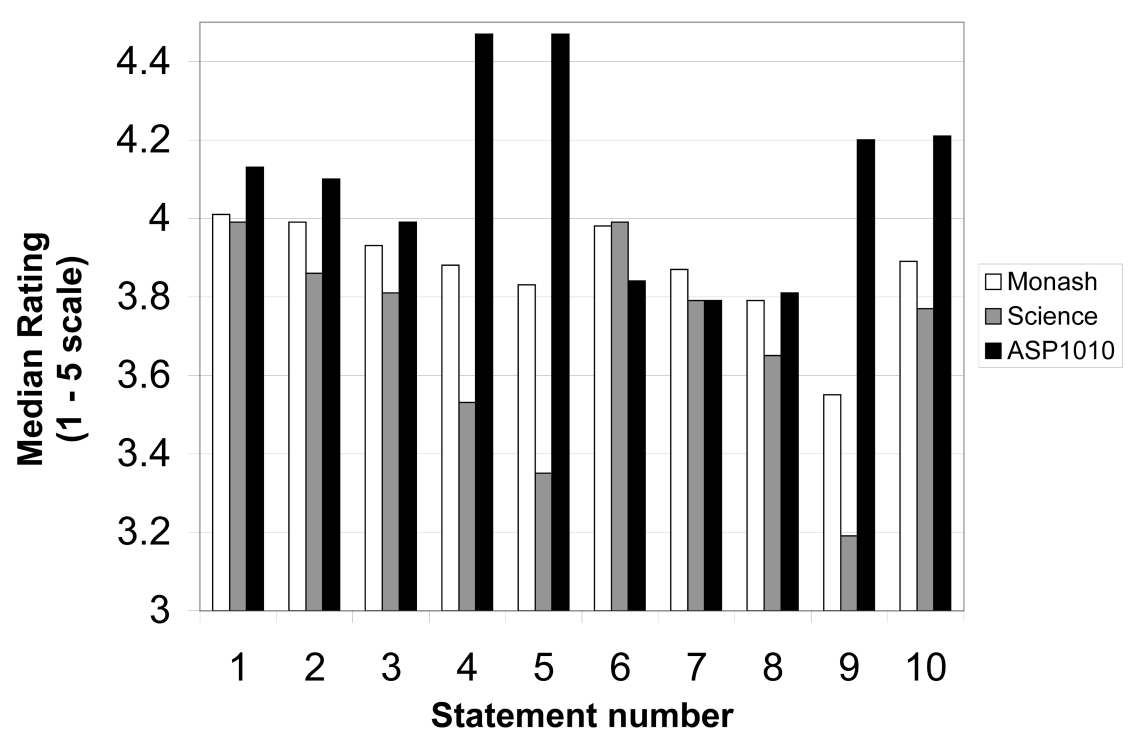

Figure 1-Median rating of students' responses to statements assessing ASP1010, compared with all Science Faculty subjects and with all Monash University subjects. Scale $1=$ 'strongly disagree' to $5=$ 'strongly agree'.

Statements:

1 The objectives of the subject were made clear.

2 The subject developed my understanding of key concepts.

3 The subject developed my skills in areas relevant to the field of study.

4 The subject was intellectually stimulating.

5 I was made aware that knowledge in the field is always changing.

6 The assessment tasks I have completed so far have helped me to learn.

7 The teaching program was well organised.

8 I received helpful feedback (e.g. in class, in discussion with staff, on assignments).

9 The subject made me want to find out more about the field.

10 Overall, I was satisfied with the quality of this subject.

\section{The 'Eyes-on' Experience}

In 1999, the second year of offering the subject, field trips were organised for groups of up to 12 students at a time to SkyBurnett in the Dandenong Ranges, an observatory operated by Dr Keith Thompson. Students were invited to attend, with up to $3 \%$ bonus marks available to those who completed and submitted an assessment sheet describing the telescope, the viewing conditions and the objects viewed.

The assessment sheet has space for further comments. Unexpectedly, many of the comments were not further information about the objects viewed but instead comments on the field trip experience. They included:

'I had the most exciting and amazing time of my life.'

'. . you rock for taking us to this. I loved it and learned a lot.'

'Amazed at the detail seen of the Moon. Excited at being able to manipulate telescope. 'Eyes-on' experience of galaxies, stars, etc. is invaluable.'
With this kind of feedback, we cannot stress too strongly the educational and motivational value of a field trip to an observatory!

\section{Subject Evaluation}

During their workshops in week 12 of semester, students were asked to complete a subject evaluation comprising 23 questions developed by the Monash University Teaching Evaluation Unit. ASP1010 rated very well; comparisons with the median ratings on ten core statements for all subjects taught in the Faculty of Science, and for all subjects taught at Monash University, are shown in Figure 1.

Students were invited to write any additional comments on the reverse side of the subject evaluation response form. These included:

'My demonstrator was excellent and I felt I got a lot out of his workshops when I asked questions about the course.'

'This subject was highly stimulating and very informative.' 
'Kept students interested and involved. Great demonstrator-interesting, enthusiastic and approachable. Great subject!!!'

'The field of study was totally irrelevant to the areas addressed in my course. Despite this I was very motivated to complete the required work and develop my understanding.'

Something for everyone. One of the subject evaluation statements was 'The amount of work required has been reasonable.' This rated a median of 4.31 on the $1-5$ scale. Clearly the weaker students were not overstretched, whilst the stronger students were not bored.

The students' evaluations indicated that we had pleased them all-that the subject did indeed cater to the aspiring astronauts as well as to the weaker students.

Teaching Staff. Another of the subject evaluation statements was 'The demonstrators gave me sufficient assistance when asked.' This statement received a stunning median rating of 4.76 on the $1-5$ scale.

Our workshop facilitators were experienced postgraduate demonstrators-clearly a key to the success of the subject. They were involved in the development and planning of each workshop activity and later in critical discussions of appropriate revisions.

\section{Conclusion}

The diversity of activities - cut and paste, simulation, planetarium, posters, debate, the night-time observatory trip-ensured that the subject was stimulating. The students' experiences in the first few weeks were quite different to those in the last few weeks. This contrasts with subjects such as Physics, Chemistry and Biology where students are generally required to attend one laboratory session per week and, while the material changes, the broad experience is the same. Students were able to take several tools home and use them for reinforcement of the concepts learned-something not normally possible in other Science subjects.

We have developed a new subject with low capital costs-our practical activities, though extremely valuable in terms of the learning associated with them, are 'cheap' in dollar terms. We have invested in ideas, and in our workshop facilitators: they were involved in the development and revision of each activity as well as in the face-to face teaching.

The subject was very well received across the broad range of strong and weak, keen and grudging students, with the workshops the major strength. They allowed weak students to succeed-the marks achieved on the assessment sheets indicated a general mastery of the understanding and skills required-while the stronger students were able to see and develop (with our fine demonstrators) further applications of each activity.

\section{Acknowledgments}

We are indebted to Keith Bambery, Marcus Thatcher, Amanda Karakas, Robert Fardon, Kirrily Rule, Tamar Greaves and Charles Morgan for their professional and enthusiastic contributions, and to Michael Morgan, John Pilbrow and Keith Thompson for their development of some activities.

\section{References}

Folkland, M., \& Ward, J. 1996, Sundials Australia, 2nd edn (Adelaide: Sundials Australia)

Giardini, W., \& Hutton, D. 1977, Aust. Science Teachers J., 23 $88-93$

Hutton, D. 1998, Aust. \& New Zealand Physicist, 36, 253

Thompson, K. 1996, Phys. Education, 31, 382 